Pacific Journal of Mathematics

REDUCTION OF TOPOLOGICAL STABLE RANK IN

Marius Dadarlat, Gabriel NaGy,
ANDRás Némethi and Cornel PaNic 


\title{
REDUCTION OF TOPOLOGICAL STABLE RANK IN INDUCTIVE LIMITS OF $C^{*}$-ALGEBRAS
}

\author{
MARIUs DĂdĂRlat, Gabriel NAGy, \\ ANDRÁs NÉmeTHI AND CORNEL PASNicu
}

\begin{abstract}
We consider inductive limits $A$ of sequences $A_{1} \rightarrow A_{2} \rightarrow \cdots$ of finite direct sums of $C^{*}$-algebras of continuous functions from compact Hausdorff spaces into full matrix algebras. We prove that $A$ has topological stable rank (tsr) one provided that $A$ is simple and the sequence of the dimensions of the spectra of $A_{i}$ is bounded. For unital $A, \operatorname{tsr}(A)=1$ means that the set of invertible elements is dense in $A$. If $A$ is infinite dimensional, then the simplicity of $A$ implies that the sizes of the involved matrices tend to infinity, so by general arguments one gets $\operatorname{tsr}\left(A_{i}\right) \leq 2$ for large enough $i$ whence $\operatorname{tsr}(A) \leq 2$. The reduction of tsr from two to one requires arguments which are strongly related to this special class of $C^{*}$-algebras.
\end{abstract}

The problem of reduction of real rank (see [6]) for these algebras was recently studied in [2] in connection with some interesting features revealed in several papers $([3],[1],[15],[5],[12],[11])$. The reduction of tsr and real rank for other classes of $C^{*}$-algebras was studied in [22], [21], [8], [24], [17], [25].

The paper consists of three sections:

1. Preliminaries and Notation

2. Local aspects of the connecting homomorphisms

3. The Main Result.

1.

1.1. For a unital $C^{*}$-algebra $A$ and a finitely generated projective $A$-module $E$, we denote by $\operatorname{End}_{A}(E)$ the algebra of $A$-linear endomorphisms of $E$ and by $\mathrm{GL}_{A}(E)$ the group of units of $\operatorname{End}_{A}(E)$. For $E=A^{n}$ we shall write $\mathrm{GL}(n, A)$ for $\mathrm{GL}_{A}\left(A^{n}\right)$ and $\mathrm{GL}^{0}(n, A)$ for the connected component of 1 . Let $\mathrm{U}(A)$ denote the unitary group of $A$ and $\mathrm{U}(n):=\mathrm{U}\left(\mathbf{C}^{n}\right)$. A selfadjoint idempotent element of a $C^{*}$-algebra will be simply called projection.

Recall some definitions from [23]. For a unital $C^{*}$-algebra $A$ and a natural number $n$ let $L g_{n}(A)$ denote the set of $n$-tuples of elements of $A$ which generate $A$ as a left ideal. The topological stable rank of $A$ is the least $n$ (if it does not exist it will be taken by definition 
to be $\infty)$ such that $\operatorname{Lg}_{n}(A)$ is dense in $A^{n}$. One denotes by $\operatorname{csr}(A)$ the least integer $n$ such that $\mathrm{GL}^{0}(m, A)$ acts transitively by right multiplication on $\operatorname{Lg}_{m}(A)$ for any $m \geq n$. (If no such integer exists one takes $\operatorname{csr}(A)=\infty$.) For nonunital $A$ one takes $\operatorname{tsr}(A):=\operatorname{tsr}(\widetilde{A})$ and $\operatorname{csr}(A):=\operatorname{csr}(\widetilde{A})$ where $\widetilde{A}$ is the algebra obtained from $A$ by adjoining a unit.

For a compact Hausdorff space $X$ of finite covering dimension one has:

$$
\begin{aligned}
& \operatorname{tsr}(C(X))=\left[\frac{\operatorname{dim} X}{2}\right]+1, \\
& \operatorname{csr}(C(X)) \leq\left[\frac{\operatorname{dim} X+1}{2}\right]+1
\end{aligned}
$$

(see [23] and [18]).

1.2. We consider $C^{*}$-inductive limits

$$
A=\lim _{\longrightarrow}\left(A_{i}, \Phi_{i j}\right) \text {. }
$$

The $A_{i}$ 's are $C^{*}$-algebras of the form

$$
A_{i}=\bigoplus_{t=1}^{s(i)} C\left(X_{i t}\right) \otimes M_{n(i, t)}
$$

where $X_{i t}$ is a Hausdorff compact space, $s(i), n(i, t)$ are positive integers and $M_{n(i, t)}$ is the $C^{*}$-algebra of complex $n(i, t) \times n(i, t)$ matrices. The $*$-homomorphisms $\Phi_{i j}: A_{i} \rightarrow A_{j}$ are not assumed to be unital or injective. We denote by $\Phi_{i}$ the natural map $A_{i} \rightarrow A$ and by $X_{i}=\bigsqcup_{t=1}^{s(i)} X_{i t}$ the spectrum of $A_{i}$.

We begin with a brief discussion on the $*$-homomorphisms between certain homogeneous $C^{*}$-algebras.

1.3. For given $C^{*}$-algebras $C, D$ we denote by $\operatorname{Hom}(C, D)$ the space of all *-homomorphisms from $C$ to $D$ with the point-norm topology. $\operatorname{Hom}^{1}(C, D)$ stands for the subspace of unital $*$-homomorphisms. We shall identify

$$
\operatorname{Hom}\left(C(X), C(Y) \otimes M_{n}\right) \quad \text { with } \operatorname{Map}\left(Y, \operatorname{Hom}\left(C(X), M_{n}\right)\right)
$$

where for topological spaces $Y, Z, \operatorname{Map}(Y, Z)$ denotes the space of continuous functions from $Y$ to $Z$ endowed with the compact-open topology.

Each $\psi \in \operatorname{Hom}\left(C(X), M_{n}\right)$ has the form

$$
\psi(f)=\sum f\left(x_{r}\right) p_{r}, \quad f \in C(X),
$$


for suitable points $x_{r} \in X$ and mutually orthogonal projections $p_{r}$ in $M_{n}$. Let $L_{\psi}$ be the set of all $x_{r}$ 's that appear in the above formula. More generally, each $\Phi \in \operatorname{Hom}\left(C(X), C(Y) \otimes M_{n}\right)$ is identified with a map $\Phi: Y \rightarrow \operatorname{Hom}\left(C(X), M_{m}\right)$ and we define for each $y \in Y$, $L_{\Phi}(y):=L_{\Phi(y)}$. In the same way for given

$$
\Phi \in \operatorname{Hom}\left(\bigoplus C\left(X_{\alpha}\right) \otimes M_{n(\alpha)}, \bigoplus C\left(Y_{\beta}\right) \otimes M_{m(\beta)}\right)
$$

and $y \in Y$ we define

$$
L_{\Phi}(y)=\bigsqcup_{\alpha} L_{\Phi_{\alpha, \beta}}(y)
$$

where $\Phi_{\alpha, \beta}$ denotes the component of $\Phi$ acting from $C\left(X_{\alpha}\right) \subset$ $C\left(X_{\alpha}\right) \otimes M_{n(\alpha)}$ to $C\left(Y_{\beta}\right) \otimes M_{m(\beta)}$.

Note that $\Phi(f)(y)=\Phi(g)(y)$ whenever $f=g$ on $L_{\Phi}(y)$.

The map $y \mapsto L_{\Phi}(y)$ has useful semicontinuity properties:

(a) if $L_{\Phi}(y)$ is contained in some open set $U$ then $L_{\Phi}(z) \subset U$ for any $z$ in some neighborhood of $y$,

(b) the set $\left\{y: L_{\Phi}(y) \cap U \neq \varnothing\right\}$ is open for each open set $U$ (see [9] and [19]).

2. We begin by giving two criteria of simplicity for $C^{*}$-algebras $A$ as above, which extend the corresponding results for AF-algebras [4] and Bunce-Deddens algebras [7].

2.1. Proposition. Let $A=\underset{\lim }{\longrightarrow}\left(A_{i}, \Phi_{i j}\right)$ be as in 1.1 and assume that the connecting homomorphisms $\Phi_{i j}$ are injective. Then the following conditions are equivalent:

(i) $A$ is simple.

(ii) For any positive integer $i$ and any open nonempty subset $U$ of $X_{i}$ there is a $j_{0}$ such that $L_{\Phi_{i j}}(x) \cap U \neq \varnothing$ for any $j \geq j_{0}$ and $x \in X_{j}$.

(iii) For any nonzero $a \in A_{i}$ there is a $j_{0}$ such that

$$
\Phi_{i j}(a)(x) \neq 0 \quad \text { for each } j \geq j_{0} \text { and } x \in X_{j} \text {. }
$$

Proof. (ii) $\Leftrightarrow$ (iii). This is clear since for given $a \in A_{i}$ one has

$$
\Phi_{i j}(a)(x)=0 \quad \text { if and only if } \quad a=0 \text { on } L_{\Phi_{i j}}(x) .
$$

(i) $\Rightarrow$ (ii). Assume that (ii) does not hold for some $i$ and some open nonempty $U \varsubsetneqq X_{i}$. Passing to a subsequence, if necessary, we may assume that for any $j \geq i$ the set $F_{j}=\left\{x \in X_{j} ; L_{\Phi_{i j}}(x) \cap U=\varnothing\right\}$ 
is nonempty and $F_{j} \neq X_{j}$. By the last part of $1.3 F_{j}$ is closed. Therefore the family $\left(J_{j}\right)_{j \geq i}$ where

$$
J_{j}=\left\{a \in A_{j}: a=0 \text { on } F_{j}\right\}
$$

defines a closed two sided ideal $J$ in $A$. (Note that $\Phi_{j k}\left(J_{j}\right) \subset J_{k}$ since $L_{\Phi_{i j}}(y) \subset L_{\Phi_{i k}}(x)$ for any $y \in L_{\Phi_{j k}}(x)$.) Also $J \neq A$ since if $e_{i}$ is the unit of $A_{i}$ then $\operatorname{dist}\left(\Phi_{i j}\left(e_{i}\right), J_{j}\right)=1$ for any $j \geq i$ and so $e_{i} \notin J$. The existence of $J$ contradicts (i).

(iii) $\Rightarrow$ (i). Let $J$ be a two-sided closed nonzero ideal of $A$. One has $\left.J=\overline{\bigcup\left(J \cap A_{i}\right.}\right)$ (see [4]). We shall prove that $J \cap A_{j}=A_{j}$ for large enough $j$. Take $a \in J \cap A_{i}, a \neq 0$. By (iii) there is a $j_{0}$ such that $\Phi_{i j}(a)(x) \neq 0$ for all $j \geq j_{0}$ and $x \in X_{j}$. Since $\Phi_{i j}\left(J \cap A_{i}\right) \subset J \cap A_{j}$ we find that $\Phi_{i j}(a) \in J \cap A_{j}$ for $j \geq j_{0}$. Since $\Phi_{i j}(a)$ does not vanish at any point of $X_{j}$ this forces $J \cap A_{j}=A_{j}$.

Let $A=\lim _{\longrightarrow}\left(A_{i}, \Phi_{i j}\right)$ be as above. For a noninvertible element $a \in A_{i}$ there are $x_{0} \in X_{i}, u \in U\left(A_{i}\right)$ and a projection $p \in A_{i}$ (both $u$ and $p$ "scalars") such that $u a\left(x_{0}\right) p=p u a\left(x_{0}\right)=0$.

For simple $A$ the following two lemmas enable us to obtain something similar for $\Phi_{i j}(a)$ (for some $j \geq i$ ) locally around any point of $X_{j}$, after a small perturbation of $a$.

2.2. Lemma. Let $\Phi \in \operatorname{Hom}\left(\bigoplus_{i=1}^{s} C\left(X_{i}\right) \otimes M_{n(i)}, C(Y) \otimes M_{m}\right)$, let $k \geq 1$, let $U$ be an open subset of $X_{1}$ and let $y \in Y$ such that $L_{\Phi}(y) \cap U$ has at least $k$ points. Then there is $p_{W} \in C(Y) \otimes M_{m}$ such that $p_{W}(z)$ is a projection of rank greater than or equal to $k$ for all $z$ in some neighborhood $W$ of $y$ and

$$
\Phi(a) p_{W}=p_{W} \Phi(a)
$$

for any $a \in \bigoplus_{i=1}^{s} C\left(X_{i}\right) \oplus M_{n(i)}$ satisfying

$$
a(x) e_{11}=e_{11} a(x)=0
$$

for all $x \in U$. (Here $\left(e_{i j}\right)$ stands for a system of matrix units of $\left.M_{n(1)} \cdot\right)$

Proof. Take $U_{1}, U_{2}$ open subsets of $X=\bigcup_{i=1}^{s} X_{i}$ having disjoint closures such that

$$
L_{\Phi}(y) \cap U \subset U_{1} \subset U, \quad L_{\Phi}(y) \cap\left(X_{1}-U\right) \subset U_{2} .
$$

Using the continuity of $L_{\Phi}$ (see 1.3) we find a neighborhood $W$ of $y$ such that $L_{\Phi}(z) \subset U_{1} \cup U_{2}$ for all $z \in W$. Take a continuous 
map $g: X_{1} \rightarrow[0,1]$ such that $g=1$ on $U_{1}$ and $g=0$ on $U_{2}$ and define $p_{W}=\Phi\left(g \otimes E_{11}\right)$. If $z \in W$ then $p_{W}(z)=p_{W}^{2}(z)=p_{W}^{*}(z)$ since $g=g^{2}=g^{*}$ on $L_{\Phi}(W)$. One has rank $p_{W}(z) \geq k$ since $L_{\Phi}(y) \cap U_{1}$ has at least $k$ elements and $g=1$ on $U_{1}$. Finally if $a(x) e_{11}=e_{11} a(x)=0$ for all $x \in U$ then $\left(g \otimes e_{11}\right) a=a\left(g \otimes e_{11}\right)=0$. This implies $p_{W} \Phi(a)=\Phi(a) p_{W}=0$.

2.3. Lemma. Let $C=C(X) \otimes M_{n}$ and let $a \in C$ such that $\operatorname{det} a(x)=0$ for some $x \in X$. Then for any $\varepsilon>0$ there exist $u$, $v \in \mathrm{GL}(C)$ and $b \in C$ such that

$\|u a v-b\|<\varepsilon \quad$ and $\quad b e_{11}=e_{11} b=0$ on a neighbourhood of $x$.

Proof. Take $u, v \in \mathrm{Gl}(n, \mathrm{C})$ such that the matrix $u a(x) v$ has only zero entries on the first row and on the first column. Now $b$ is easily found since continuous functions vanishing at $x$ can be uniformly approximated by continuous functions vanishing on a neighbourhood of $x$.

3. The next step toward the main result is based on the following theorem which follows from Michael's paper [16].

3.1. THEOREM. Let $X$ be a Hausdorff compact space of dimension $d$, let $T$ be a complete metric space and let $Y$ be a map from $X$ to the family of the nonempty closed subsets of $T$.

Suppose that

(a) $Y$ is lower semicontinuous, i.e. for each open subset $U$ of $T$ the set $\{x \in X: Y(x) \cap U \neq \varnothing\}$ is open;

(b) Each $Y(x)$ is $(d+1)$-connected;

(c) There is an $\varepsilon>0$ such that for any $0<r<\varepsilon$ and $x \in X$ the intersection of $Y(x)$ with any closed ball of radius $r$ in $T$ is a contractible space.

Then there is a continuous map $\sigma: X \rightarrow T$ such that $\sigma(x) \in Y(x)$ for all $x \in X$.

Proof. The theorem follows from Theorem 1.2 in [16] using the comments from the second part of the same paper.

3.2. Proposition. Let $X$ be a Hausdorff compact space, let $k^{\prime} \geq$ $k \geq 1$ integers, let $\mathscr{W}$ be an open cover of $X$ and assume that for each $W \in \mathscr{W}$ there is given a continuous projection valued map $p_{W}: W \rightarrow$ $M_{n}$ such that $\operatorname{rank} p_{W}(x) \geq k^{\prime}$ for $x \in W$. If $\operatorname{dim}(X) \leq 2\left(k^{\prime}-k\right)-1$ 
then there is a continuous projection valued map $p: X \rightarrow M_{n}$ such that for $x \in X$ :

$$
\begin{gathered}
\operatorname{rank} p(x) \geq k, \\
p(x) \leq \bigvee\left\{p_{W}(x): W \in \mathscr{W}, x \in W\right\}
\end{gathered}
$$

Proof. For $x \in X$ define $\mathscr{W}(x)=\{W \in \mathscr{W}: x \in W\}$ and $H(x)=$ $\operatorname{span}\left\{p_{W}(x) \mathbf{C}^{n}: W \in \mathscr{W}(x)\right\}$.

For any linear subspace $H$ of $\mathbf{C}^{n}$ let $V(H, k), k \leq \operatorname{dim}(H)$, denote the Stiefel manifold of $k$-orthogonal frames in $H$ (see [14]). For any $x \in X$ define $Y(x)=V(H(x), k) \subset V\left(\mathbf{C}^{n} ; k\right)$. We check that $Y$ satisfies the conditions of Theorem 3.1.

(a) The lower semicontinuity of $Y$ follows from the lower semicontinuity of the map $x \mapsto H(x) \subset \mathbf{C}^{n}$ which is almost obvious having in mind the definition of $H(x)$.

(b) $V(H, k)$ is $2(\operatorname{dim}(H)-k)$-connected (see [14]). Therefore $V(H(x), k)$ is $2\left(k^{\prime}-k\right)$-connected since $\operatorname{dim} H(x) \geq k^{\prime}$.

(c) For any $m, n \geq m \geq k$, there is $\varepsilon_{m}>0$ such that any closed ball of radius at most $\varepsilon_{m}$ in $V\left(\mathbf{C}^{m}, k\right)$ is contractible. (We consider $V\left(\mathbf{C}^{m}, k\right)$ with the metric induced by the restriction of a $\mathrm{U}(n)$-invariant Riemann structure on $V\left(C^{n}, k\right)$.) In this situation $V\left(\mathbf{C}^{m}, k\right)$ is a totally geodesic submanifold of $V\left(\mathbf{C}^{n}, k\right)$ and the same is true for any $V(H, k)$ with $H \subset \mathbf{C}^{n}$. Therefore the induced metric form from $V\left(\mathbf{C}^{n}, k\right)$ coincides with the metric given by the induced Riemann structure of $V(H, k)$ (see [13]). Having also the $\mathrm{U}(n)$-invariance of this metric one can take

$$
\varepsilon=\min \left\{\varepsilon_{m}: k \leq m \leq n\right\} .
$$

We also need the following approximation results:

\subsection{Lemma. Let $B$ be a unital $C^{*}$-algebra and let}

$$
k \geq \max (\operatorname{tsr}(B), \operatorname{csr}(B)) .
$$

Then for any positive integer $m$ and any $a \in M_{m}(B)$, the matrix $\left(\begin{array}{ll}a & 0 \\ 0 & 0\end{array}\right)$ belongs to the closure of $\mathrm{GL}(m+k, B)$.

Proof. If $m \leq k$ one can take

$$
b_{\varepsilon}=\left(\begin{array}{ccc}
a & \varepsilon 1_{m} & 0 \\
\varepsilon 1_{m} & 0_{m} & 0 \\
0 & 0 & \varepsilon 1_{k-m}
\end{array}\right) \in \mathrm{GL}(m+k, B)
$$

and $b_{\varepsilon} \rightarrow a$ as $\varepsilon \rightarrow 0$. 
For $m \geq k$ we proceed by induction. Assume the statement holds for a fixed $m \geq k$ and let a $a \in M_{m+1}(B)$. Since

$$
m \geq \max (\operatorname{tsr}(B), \operatorname{csr}(B))
$$

it follows from [23] that for each $\varepsilon>0$ there are $t \in \mathrm{GL}(m+1, B)$, $a_{1} \in M_{m}(B)$ and $b \in B^{m}$ such that

$$
\left\|a-\left(\begin{array}{cc}
1 & 0 \\
b & a_{1}
\end{array}\right) \cdot t\right\|<\varepsilon .
$$

By the induction hypothesis one can approximate

$$
\left(\begin{array}{ccc}
1 & 0 & 0 \\
b & a_{1} & 0 \\
0 & 0 & 0_{k}
\end{array}\right)
$$

with an invertible matrix of the form

$$
\left(\begin{array}{l|ll}
1 & 0 & 0 \\
b & c
\end{array}\right)
$$

Hence $\left(\begin{array}{ll}a & 0 \\ 0 & 0 \\ k\end{array}\right)$ will be approximated by

$$
\left(\begin{array}{c|cc}
1 & 0 & 0 \\
b & c
\end{array}\right) \cdot\left(\begin{array}{cc}
t & 0 \\
0 & 1_{k}
\end{array}\right) .
$$

3.4. Remark. Suppose $B, k$ are as above. Let $F, G, H$ be finitely generated projective $B$-modules and put $E=F \oplus G \oplus H$. If $F, G$ are free and $G \simeq B^{k}$, then a slight modification of the above arguments shows that $\operatorname{End}_{B}(F) \subset \overline{\mathrm{GL}_{B}(E)}$.

In the proof of the main result we shall invoke the following straightforward approximation device:

3.5. LEMMA. Let $B=\overline{U B_{i}}$ where the $B_{i}$ 's form an increasing sequence of unital $C^{*}$-algebras. Let $e_{i}$ be the unit of $B_{i}$. If for any $a \in B_{i}$ and $\varepsilon>0$ there is $j \geq i$ and $b \in \mathrm{GL}\left(e_{i} B_{j} e_{i}\right)$ such that $\|a-b\|<\varepsilon$ then $\operatorname{tsr}(B)=1$.

Proof. Let $\widetilde{B}=B+C \cdot 1$ be the algebra obtained by adjoining a unit to $B$. Let $x+\lambda 1 \in \widetilde{B}$ with $x \in B_{i}$. By hypothesis there is $j \geq i$ and $y \in \mathrm{GL}\left(e_{i} B_{j} e_{i}\right) \subset \mathrm{GL}\left(e_{i} B e_{i}\right)$ such that $\left\|x+\lambda e_{i}-y\right\|$ is small. Choosing a non zero scalar $\lambda^{\prime}$ close to $\lambda$, the element $y+\lambda^{\prime}\left(1-e_{i}\right)$ is invertible and approximates $x+\lambda \cdot 1$. Therefore $\operatorname{GL}(\widetilde{B})$ is dense in $\widetilde{B}$ which means $\operatorname{tsr}(B)=1$. 
3.6. Theorem. Let $A=\lim _{\longrightarrow}\left(A_{i}, \Phi_{i j}\right)$ where $A_{i}=\bigoplus_{t=1}^{s(i)} C\left(X_{i t}\right) \otimes$ $M_{n(i, t)}$, each $X_{i t}$ being a Hausdorff compact space such that $d=$ $\sup \operatorname{dim}\left(X_{i t}\right)<\infty$.

If $A$ is simple then $\operatorname{tsr}(A)=1$.

Proof. Replacing each $A_{i}$ by its image in $A$ one may suppose that all the $\Phi_{i j}$ 's are injective. We shall verify the conditions from Lemma 3.5. Let $a \in A_{i}$ be a noninvertible element and put $Z=\{x \in$ $\left.X_{i}: \operatorname{det} a(x)=0\right\}$. If $Z$ consists only of isolated points of $X_{i}$ then it is obvious that $a \in \overline{\mathrm{GL}\left(A_{i}\right)}$. Thus we may assume that there is $x \in Z$ such that each neighbourhood of $x$ is an infinite set.

Moreover by Lemma 2.3 we may suppose that $a e_{11}^{t}=e_{11}^{t} a=0$ on some neighbourhood $U$ of $x$ for some $t$. Fix integers $k^{\prime}, k$ such that

$$
k \geq 2 d+4, \quad 2\left(k^{\prime}-k\right)+1 \geq d .
$$

Since $U$ is an infinite open set and the $C^{*}$-algebra $A$ is simple it follows by Proposition 2.1 that there is $j \geq i$ such that $L_{\Phi_{i j}}(y) \cap U$ has at least $k^{\prime}$ elements for any $y \in X_{j}$. This enables us by using Lemma 2.2 to find an open covering $\mathscr{W}$ of $X_{j}$ such that for each $W \in \mathscr{W}$ there is $p_{W} \in A_{j}$ satisfying

(1) $p_{W}$ is projection valued on $W$,

(2) rank $p_{W}(y) \geq k^{\prime}$ for any $y \in W$,

(3) $p_{W} \Phi_{i j}(a)=\Phi_{i j}(a) p_{W}=0$ on $W$,

(4) $p_{W} \leq \Phi_{i j}\left(e_{i}\right)$ where $e_{i}$ is the unit of $A_{i}$.

Proposition 3.2 provides us a projection $p \in A_{j}$ such that

(a) $p(x) \leq \bigvee\left\{p_{W}(x): W \in \mathscr{W}, x \in W\right\}$ for all $x \in X_{j}$.

(b) $\operatorname{rank} p(x) \geq k$ for all $x \in X_{j}$.

Of course (4) and (a) imply that $p \leq \Phi_{i j}\left(e_{i}\right)$.

We have also

(c) $\Phi_{i j}(a) p=p \Phi_{i j}(a)=0$

as a consequence of (3) and (a).

Let $b:=\Phi_{i j}(a)$ have the components $\left(b_{t}\right)$ with $b_{t} \in C\left(X_{j t}\right) \otimes$ $M_{n(j, t)}$. We shall use Remark 3.4 in order to approximate each $b_{t}$ by invertible elements in $\operatorname{End}_{C\left(X_{j t}\right)}\left(E_{t}\right)$ where $E_{t}:=\Phi_{i j}\left(e_{i}\right) C\left(X_{j t}\right)^{n(j, t)}$. Consider also the finitely generated projective $C\left(X_{j t}\right)$-modules

$$
P_{t}=p C\left(X_{j t}\right)^{n(j, t)}, \quad Q_{t}=\left(\Phi_{i j}\left(e_{i}\right)-p\right) C\left(X_{j t}\right)^{n(j, t)} .
$$

It is clear that $E_{t} \simeq P_{t} \oplus Q_{t}$.

Since rank $P_{t} \geq k \geq 2 d+4$, by using the stability properties of vector bundles (see [14]), one can split $P_{t}$ as a direct sum of finitely 
generated projective $C\left(X_{j t}\right)$-modules $P_{t}=R_{t} \oplus G_{t} \oplus H_{t}$ such that $Q_{t} \oplus R_{t}$ and $G_{t}$ are free and

rank $G_{t} \geq[(d+1) / 2]+1 \geq \max \left\{\operatorname{tsr} C\left(X_{j t}\right), \operatorname{csr} C\left(X_{j t}\right)\right\}$.

Let $F_{t}=Q_{t} \oplus R_{t} \oplus G_{t}$. By equation (c) above one can regard $b_{t}$ as an element of $\operatorname{End}_{C\left(X_{j t}\right)}\left(F_{t}\right)$ that vanishes on $G_{t}$. Since both $F_{t}$ and $G_{t}$ are free it follows from Lemma 3.3 that $b_{t}$ belongs to the closure of $\mathrm{GL}\left(F_{t}\right)$. As $F_{t}$ is a direct summand in $E_{t}$, this implies that $b_{t}$ belongs to the closure $\mathrm{GL}\left(E_{t}\right)$. It follows that $\Phi_{i j}(a)$ belongs to the closure of $\mathrm{GL}\left(\bigoplus_{t} E_{t}\right)=\mathrm{GL}\left(\Phi_{i j}\left(e_{i}\right) A_{j} \Phi_{i j}\left(e_{i}\right)\right)$. The proof is complete by virtue of Lemma 3.5.

\section{REFERENCES}

[1] B. Blackadar, Symmetries of the CAR algebra, preprint, 1988.

[2] B. Blackadar, O. Brattelli, G. A. Elliott and A. Kumjian, Reduction of real rank and inductive limits of $C^{*}$-algebras, preprint.

[3] B. Blackadar and A. Kumjian, Skew products of relations and the structure of simple $C^{*}$-algebras, Mat. Z., 189 (1985), 55-63.

[4] O. Brattelli, Inductive limits of finite-dimensional $C^{*}$-algebras, Trans. Amer. Math. Soc., 171 (1972), 195-234.

[5] O. Brattelli, G. A. Elliott, D. E. Evans and A. Kishimoto, Finite group actions on AF algebras obtained by folding the interval, preprint, 1989.

[6] L. B. Brown and G. K. Pedersen, $C^{*}$-algebras of real rank zero, preprint, 1989.

[7] J. Bunce and J. Deddens, A family of simple $C^{*}$-algebras related to weighted shift operators, J. Funct. Anal., 19 (1975), 12-34.

[8] M.-D. Choi and G. A. Elliott, Density of the self-adjoint elements with finite spectrum in an irrational rotation $C^{*}$-algebra, preprint, 1988.

[9] M. Dădărlat, On homomorphisms of certain $C^{*}$-algebras, preprint, 1986.

[10] M. Dădărlat and A. Némethi, Shape theory and connective $K$-theory, to appear in J. Operator Theory.

[11] G. A. Elliott, On the classification of $C^{*}$-algebras of real rank zero, preprint.

[12] D. E. Evans and A. Kishimoto, Compact group actions on UHF algebras obtained by folding the interval, J. Funct. Anal., (to appear).

[13] S. Helgason, Differential Geometry, Lie Groups and Symmetric Spaces, Academic Press, 1978.

[14] D. Husemoller, Fibre Bundles, 2nd ed., Springer Verlag, 1966.

[15] A. Kumjian, An involutive automorphism of the Bunce-Deddens algebra, C.R. Math. Rep. Acad. Sci. Canada, 10 (1988), 217-218.

[16] E. Michael, Continuous selections II, Ann of Math., 64, no. 3, (1956), 562-580.

[17] G. Nagy, Some remarks on lifting invertible elements from quotient $C^{*}$-algebras, J. Operator Theory, 21 (1989), 379-386.

[18] V. Nistor, Stable range for tensor products of extensions of $\mathscr{K}$ by $C(X), \mathrm{J}$. Operator Theory, 16 (1986), 387-396.

[19] C. Pasnicu, On inductive limits of certain $C^{*}$-algebras of the form $C(X) \otimes F$, Trans. Amer. Math. Soc., 310 (1988), 703-714.

[20] G. K. Pedersen, $C^{*}$-algebras and their Automorphism Groups, Academic Press, London/New York, 1979. 
[21] I. F. Putnam, The invertible elements are dense in the irrational rotation $C^{*}$ algebras, preprint, 1989.

[22] N. Riedel, On the topological stable rank of irrational rotation algebras, J. Operator Theory, 13 (1985), 143-150.

[23] M. A. Rieffel, Dimension and stable rank in the $K$-theory of $C^{*}$-algebras, Proc. London Math. Soc., 46 (1983), 301-333.

[24] M. Rordam, On the structure of simple $C^{*}$-algebras tensored with a UHF-algebra I, II, preprints.

[25] S. Zhang, $C^{*}$-algebras with real rank zero and the internal structure of their corona and multiplier algebras I, II, III, IV, preprints.

Received October 5, 1990 and in revised form June 7, 1991.

UNIVERSITY OF CALIFORNIA

Los ANGeles, CA 90024-1555

UNIVERSITY OF CALIFORNIA

BERKELEY, CA 94720

Ohio State University

Columbus, OH 43210

AND

INSTITUTE OF MATHEMATICS

BD. PACII 220

79622 BUCHAREST, ROMANIA 


\section{PACIFIC JOURNAL OF MATHEMATICS EDITORS}

\author{
V. S. VARADARAJAN \\ (Managing Editor) \\ University of California \\ Los Angeles, CA 90024-1555-05 \\ Herbert Clemens \\ University of Utah \\ Salt Lake City, UT 84112 \\ F. Michael Christ \\ University of California \\ Los Angeles, CA 90024-1555 \\ THOMAS ENRIGHT \\ University of California, San Diego \\ La Jolla, CA 92093
}

Nicholas ERCOLANI

University of Arizona

Tucson, AZ 85721

R. FINN

Stanford University

Stanford, CA 94305

VAUGHAN F. R. JONES

University of California

Berkeley, CA 94720

Steven Kerckhoff

Stanford University

Stanford, CA 94305

\section{C. MOORE}

University of California

Berkeley, CA 94720

Martin ScharlemanN

University of California

Santa Barbara, CA 93106

HAROLD STARK

University of California, San Diego

La Jolla, CA 92093

\section{R. ARENS \\ ASSOCIATE EDITORS}

\begin{tabular}{|c|c|c|c|c|}
\hline R. ARENS & $\begin{array}{l}\text { E. F. BECKENBACH } \\
(1906-1982)\end{array}$ & B. H. NeumanN & $\begin{array}{c}\text { F. WolF } \\
(1904-1989)\end{array}$ & K. YoshidA \\
\hline \multicolumn{5}{|c|}{ SUPPORTING INSTITUTIONS } \\
\hline \multicolumn{2}{|c|}{ UNIVERSITY OF ARIZONA } & UNIVER: & OF OREG & \\
\hline \multicolumn{2}{|c|}{ UNIVERSITY OF BRITISH COLUMB } & & & \\
\hline \multicolumn{2}{|c|}{ CALIFORNIA INSTITUTE OF TECHNOLOGY } & OGY STANI & INIV & \\
\hline \multicolumn{2}{|c|}{ UNIVERSITY OF CALIFORNIA } & UNI & & \\
\hline \multicolumn{2}{|c|}{ MONTANA STATE UNIVERSITY } & UNIVE & OF T & \\
\hline \multirow{2}{*}{\multicolumn{2}{|c|}{ NIVERSITY OF NEVADA, RENO }} & UNIVE & OF U & \\
\hline & & WASH & N ST & ERSIT \\
\hline \multicolumn{2}{|c|}{$\begin{array}{l}\text { NEW MEXICO STATE UNIVERSITY } \\
\text { OREGON STATE UNIVERSITY }\end{array}$} & UNIVERS & OF WASHI & \\
\hline
\end{tabular}

The Supporting Institutions listed above contribute to the cost of publication of this Journal, but they are not owners or publishers and have no responsibility for its content or policies.

Mathematical papers intended for publication in the Pacific Journal of Mathematics should be in typed form or offset-reproduced (not dittoed), double spaced with large margins. Please do not use built up fractions in the text of the manuscript. However, you may use them in the displayed equations. Underline Greek letters in red, German in green, and script in blue. The first paragraph must be capable of being used separately as a synopsis of the entire paper. In particular it should contain no bibliographic references. Please propose a heading for the odd numbered pages of less than 35 characters. Manuscripts, in triplicate, may be sent to any one of the editors. Please classify according to the 1991 Mathematics Subject Classification scheme which can be found in the December index volumes of Mathematical Reviews. Supply name and address of author to whom proofs should be sent. All other communications should be addressed to the managing editor, or Elaine Barth, University of California, Los Angeles, California 90024-1555-05.

There are page-charges associated with articles appearing in the Pacific Journal of Mathematics. These charges are expected to be paid by the author's University, Government Agency or Company. If the author or authors do not have access to such Institutional support these charges are waived. Single authors will receive 50 free reprints; joint authors will receive a total of 100 free reprints. Additional copies may be obtained at cost in multiples of 50 .

The Pacific Journal of Mathematics (ISSN 0030-8730) is published monthly except for July and August. Regular subscription rate: $\$ 190.00$ a year (10 issues). Special rate: $\$ 95.00$ a year to individual members of supporting institutions.

Subscriptions, orders for numbers issued in the last three calendar years, and changes of address should be sent to Pacific Journal of Mathematics, P.O. Box 969, Carmel Valley, CA 93924, U.S.A. Old back numbers obtainable from Kraus Periodicals Co., Route 100, Millwood, NY 10546.

The Pacific Journal of Mathematics at P.O. Box 969, Carmel Valley, CA 93924 (ISSN 0030-8730) is published monthly except for July and August. Second-class postage paid at Carmel Valley, California 93924, and additional mailing offices. Postmaster: send address changes to Pacific Journal of Mathematics, P.O. Box 969, Carmel Valley, CA 93924.

PUBLISHED BY PACIFIC JOURNAL OF MATHEMATICS, A NON-PROFIT CORPORATION Copyright (C) 1992 by Pacific Journal of Mathematics 


\section{Pacific Journal of Mathematics}

Vol. 153, No. $2 \quad$ April, 1992

R. Ayala, Eladio Domínguez Murillo, Alberto Márquez Pérez and A.

Quintero, Lusternik-Schnirelmann invariants in proper homotopy

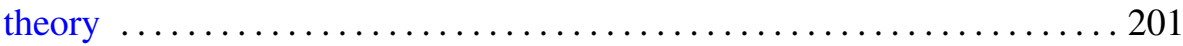

Hari Bercovici and Dan-Virgil Voiculescu, Lévy-Hinčin type theorems for multiplicative and additive free convolution $\ldots \ldots \ldots \ldots \ldots \ldots \ldots 217$

L. J. Bunce and Cho-Ho Chu, Compact operations, multipliers and Radon-Nikodým property in $J B^{*}$-triples $\ldots \ldots \ldots \ldots \ldots \ldots \ldots \ldots \ldots \ldots \ldots \ldots$

Marius Dadarlat, Gabriel Nagy, András Némethi and Cornel Pasnicu, Reduction of topological stable rank in inductive limits of $C^{*}$-algebras

François Dumas and Robert Vidal, Dérivations, et hautes dérivations, dans certains corps gauches de series de Laurent .................... 277

Mourad Ismail and Xin Li, On sieved orthogonal polynomials. IX:

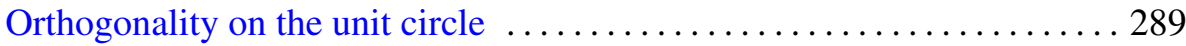

X. T. Liang and Y. W. Lu, A Phragmén-Lindelöf theorem ...............299

Mark Stephen Reeder, On certain Iwahori invariants in the unramified principal series

Shohei Tanaka, On the representation of the determinant of Harish-Chandra's $C$-function of $\operatorname{SL}(n, \mathbb{R})$

Fritz von Haeseler and Guentcho Svetoslavov Skordev, Borsuk-Ulam theorem, fixed point index and chain approximations for maps with multiplicity 